Original Article

\title{
Measuring cerebral hemodynamic changes during action observation with functional transcranial doppler
}

\author{
SeOng-Sik Kim, PT, PhD ${ }^{1)}$, Byoung-Hee Lee, PT, $\mathrm{PhD}^{1)^{*}}$ \\ 1) Department of Physical Therapy, Sahmyook University: 815 Hwarang-ro, Nowon-gu, Seoul 139-742, \\ Republic of Korea
}

\begin{abstract}
Purpose] The purpose of this study was to investigate the effects of action observation training (AOT) on cerebral hemodynamic changes including cerebral blood flow velocity (CBFV) and cerebral blood flow volume (CBFvol) in healthy subjects. [Subjects] Fifteen healthy subjects participated in this study. [Methods] All subjects were educated regarding AOT, and systolic peak velocity (Vs) as well as mean flow velocity $(\mathrm{Vm})$ in the middle cerebral artery (MCA), anterior cerebral artery (ACA), and posterior cerebral artery (PCA) were evaluated using functional transcranial doppler with a 2-MHz probe, before and after performing AOT. [Results] Healthy subjects showed significant differences in Vs and Vm in the MCA, ACA, and PCA after AOT compared with those before AOT. [Conclusion] Our findings indicate that AOT has a positive effect in terms of an increase in CBFV and CBFvol in healthy subjects, since the brain requires more blood to meet the metabolic demand during AOT. Key words: Action observation, Cerebral blood flow, Functional transcranial doppler
\end{abstract}

(This article was submitted Dec. 5, 2014, and was accepted Jan. 11, 2015)

\section{INTRODUCTION}

Therapeutic applications that engage the mirror system, including action observation training (AOT), motor imagery, and imitation, have been suggested to help improve objective programming during stroke rehabilitation ${ }^{1)}$. AOT includes understanding the movement, imitation learning, motor learning, and cognitive-action processing ${ }^{2-4}$ such as motor memory formation by providing distinct and concrete standard motions, which enable clear motor imagery ${ }^{5)}$ to engage the motor neural network in the same manner as motor execution and action observation ${ }^{6}$. AOT increases excitability of the primary motor cortex involved in cognitive processing, which is related to kinesthetic memory formation, motor learning, imitation learning, and understanding the purpose of the movement ${ }^{7}$. However, it can be difficult for subjects to imagine the entire process while an exact motor representation of the task is being performed ${ }^{8)}$.

The mu rhythm that synchronizes motor observation, motor imagery, and motor execution is generally activated within a frequency of $8-13 \mathrm{~Hz}$ through the sensory motor cortex $^{9)}$, which is related to increased excitability of the primary motor cortex. $\mathrm{Mu}$ desynchronization, on the other hand, is related to the premotor cortex mirror neuron sys-

*Corresponding author. Byoung-Hee Lee (E-mail: 3679@syu. ac.kr)

(C2015 The Society of Physical Therapy Science. Published by IPEC Inc. This is an open-access article distributed under the terms of the Creative Commons Attribution Non-Commercial No Derivatives (by-ncnd) License $<$ http://creativecommons.org/licenses/by-nc-nd/3.0/>. tem ${ }^{10)}$. These specific neurophysiologic signs are activated when observing or imagining the moving and stopping of oneself or others ${ }^{11)}$.

Cerebral function and metabolism are strongly associated with cerebral blood flow (CBF), since an increase in the metabolic demand in the brain leads to an increase in blood flow ${ }^{12)}$. Therefore, neuronal activity is coupled with the blood supply in the brain ${ }^{13)}$.

Recently, transcranial Doppler (TCD) ultrasonography has been increasingly employed as a non-invasive, inexpensive, safe, and portable technique for measuring cerebrovascular function. It permits continuous and bilateral recording of cerebral blood flow velocity (CBFV) through the major intracranial vessels ${ }^{14,15)}$. It is also relatively resistant to movement artifacts and has good test-retest reliability ${ }^{16)}$. TCD can be performed easily, and has become an important alternative for quantifying $\mathrm{CBF}$ changes that accompany cerebral activity in the circulation of the human brain ${ }^{17)}$, and for evaluating changes in $\mathrm{CBFV}$ at rest and during performance of cognitive tasks ${ }^{14,18)}$.

Given that the contribution of cerebrovascular function to cognition is important, the purpose of this study was to investigate the effects of AOT on cerebral hemodynamic changes, including $\mathrm{CBFV}$, in healthy subjects.

\section{SUBJECTS AND METHODS}

This study had a cross-sectional design. Fifteen healthy subjects without any pre-existing neurologic or orthopedic disorder were included in this study. General characteristics of the subjects were as follows: mean age, $58.0 \pm 12.05$ years; mean height, $166.33 \pm 5.62 \mathrm{~cm}$; and mean weight, $63.93 \pm$ 
1380 J. Phys. Ther. Sci. Vol. 27, No. 5, 2015

Table 1. Comparison of cerebral hemodynamic changes $(\mathrm{N}=15)$

\begin{tabular}{lccccccc}
\hline & \multicolumn{2}{c}{ MCA } & \multicolumn{2}{c}{ ACA } & \multicolumn{2}{c}{ PCA } \\
\hline & & Rt & Lt & Rt & Lt & Rt & Lt \\
\hline \multirow{2}{*}{ Vs } & rest & $99.5 \pm 28.5$ & $97.7 \pm 28.0$ & $74.6 \pm 14.9$ & $85.5 \pm 26.3$ & $35.9 \pm 4.0$ & $37.0 \pm 4.1$ \\
& AO & $103.3 \pm 28.8^{* *}$ & $101.3 \pm 27.7^{* *}$ & $76.9 \pm 15.5^{* *}$ & $89.5 \pm 29.0^{* *}$ & $38.0 \pm 3.9^{* * *}$ & $38.3 \pm 5.5$ \\
\multirow{2}{*}{ Vm } & rest & $67.3 \pm 17.7$ & $66.7 \pm 16.8$ & $50.4 \pm 11.1$ & $58.0 \pm 17.2$ & $25.3 \pm 2.5$ & $26.1 \pm 3.9$ \\
& AO & $70.7 \pm 16.8^{* *}$ & $69.5 \pm 16.4^{* * *}$ & $52.0 \pm 10.9^{* *}$ & $60.9 \pm 18.3^{* *}$ & $26.3 \pm 2.9^{*}$ & $26.8 \pm 3.8$ \\
\hline
\end{tabular}

${ }^{\mathrm{a} M}$ (SD), Vs: systolic peak velocity, Vm: mean flow velocity, AO: action observation, MCA: middle cerebral artery, ACA: anterior cerebral artery, PCA: posterior cerebral artery, ${ }^{*} \mathrm{p}<0.05$ from rest, ${ }^{* *} \mathrm{p}<0.01$ from rest, ${ }^{* * *} \mathrm{p}<0.001$ from rest

$8.01 \mathrm{~kg}$. The objective of the study and its requirements were explained to all subjects, who signed a written informed consent form before participating in the experiment. This study was approved by the Ethics Committee of our institution (KOMCIRB-2013-50).

AOT tasks consisting of 18 different vital actions for daily living, which gradually increased in difficulty and complexity were presented. Each task was $10 \mathrm{~s}$ long, for a total task clip time of $3 \mathrm{~min}$. The participants observed and performed the 18 tasks with their own hands. During the 3-min task clip, the subjects sat comfortably in a chair with their arms placed on a table, and were asked to carefully observe 18 different video sequences of functional movements that were required in their daily lives. Each sequence became increasingly difficult (starting with turning a faucet, and then performing more complex movements such as tightening shoelaces). All participants had a 5-min rest period before imitating the motor activities and undergoing functional transcranial Doppler (fTCD) ultrasonography.

TCD ultrasonography was conducted before and after AOT using the PMD-150 system (Medasonics, USA), with the subjects lying supine on a bed, in place with a head frame, in a quiet laboratory. A 2-Mhz probe was used to measure the systolic peak velocity (Vs) with a pulse repetition frequency of $2-12 \mathrm{kHz}$, depth setting of $16-150 \mathrm{~mm}$, burst width of $6-12 \mu / \mathrm{s}$, power value of $10-100 \mathrm{~mW} / \mathrm{cm}^{2}$, and scale setting of $0-40 \mathrm{~cm} / \mathrm{s}$ for the low range, and $0-320 \mathrm{~cm} / \mathrm{s}$ for the high range. Vs was evaluated in the middle cerebral artery (MCA), anterior cerebral artery (ACA), and posterior cerebral artery (PCA). A transtemporal approach using a 2-MHz probe attached to a headpiece was used for TCD mapping of the cerebral artery to locate each blood vessel based on the flow direction, depth, and mapping display. Mean flow velocity $(\mathrm{Vm})$ in the MCA, ACA, and PCA in $\mathrm{cm} / \mathrm{s}$ was determined by dividing the maximum and minimum TCD frequencies. All measurements were repeated 3 times in random order. Offline analysis was conducted to assess the measurement data saved in HDD.

SPSS version 19.0 (IBM Corp., Armonk, NY, USA) was used for statistical analyses. The Shapiro-Wilk test was used to determine the distributions of the general properties and outcome measures of the subjects. The paired t test was used to compare cerebral hemodynamics before AOT and after AOT within each group, whereas the independent $t$ test was performed to compare the right and left sides. A $\mathrm{p}$ value $<0.05$ was considered significant.

\section{RESULTS}

Vs in the right MCA (RMCA) and left MCA (LMCA) was significantly increased after AOT compared with that before AOT. Vm in the RMCA and LMCA was also significantly increased after AOT compared with that before AOT. Vs in the right ACA (RACA) and left ACA (LACA) was significantly increased after AOT compared with that before AOT. Vm in the RACA and LACA was also significantly increased after AOT compared with that before AOT. Vs in the right PCA (RPCA) and left PCA (LPCA) was significantly increased after AOT compared with that before AOT. Vm in the RPCA and LPCA was also significantly increased after AOT compared with that before AOT. In a comparison of the MCA, ACA, and PCA using the independent t-test, there were no significant differences in $\mathrm{Vs}$ and $\mathrm{Vm}$ between the right and left sides (Table 1).

\section{DISCUSSION}

This study demonstrated the effects of AOT on cerebral hemodynamics, including $\mathrm{Vs}$ and $\mathrm{Vm}$, in the MCA, ACA, and PCA of healthy subjects. An increase in CBF is important to satisfy the metabolic demands of the cerebral nerve cell during brain activity ${ }^{19)}$, and an increase in brain metabolism can be referred to as an increase in blood flow in the internal carotid artery and $\mathrm{MCA}^{19,20)}$. We observed significant increases in $\mathrm{CBFV}$ and cerebral blood flow volume (CBFvol) in the MCA, ACA, and PCA after AOT compared with those before AOT in these healthy subjects. These findings indicate that AOT has a positive influence in terms of an increase in CBFV and CBFvol, since the brain requires more blood to meet the metabolic demand. Measurement of blood flow velocity in the main arteries using fTCD has been accepted in a variety of studies for analyzing brain activities during the performance of cognitive tasks ${ }^{14,18}$.

Previous studies have investigated the relationship between cerebral hemodynamic changes and the performance of mental tasks using fTDC, and have suggested that fTDC, as a noninvasive device, can provide quantitative and qualitative measures of psychological parameters ${ }^{18)}$. AOT is a rehabilitation method based on mirror neurons and exercise observed motion after action observation. Faster recovery of motor function is possible by observing the movement before it is performed, since observation activates the mirror neurons ${ }^{21)}$. Cognition can be assessed by comparing Vs and $\mathrm{Vm}$ in the $\mathrm{MCA}^{22)}$. $\mathrm{Koch}^{23)}$ reported that during active exer- 
cise of the arm, Vs in the MCA was increased. Thus, active exercise plays a great role in increasing the blood flow in the brain. In Sato's study regarding motion observation, it was revealed that an increase in MCA blood flow also increased the afferent input of muscle spindles and joint receptors, activating a wide cerebral area, including the supplementary motor area, after transferring to the somatosensory area of the cerebrum through the thalamus, which controls general movement. Activation of this area indicates that the mirror neuron system is activated, which involves the same brain function involved in performing the motion in reality ${ }^{24)}$. This study confirmed that AOT can contribute to cerebral hemodynamic changes and enhance cerebral metabolism, since it increased Vs and Vm. Therefore, exercise after action observation is expected to be more effective for motor learning, since it activates the mirror neurons that cause a motor reaction in the same manner as actual exercise. The frontal lobe and frontal supplementary motor area are activated in the early stage of motor learning followed by the parietal lobe. This study demonstrated that AOT increased $\mathrm{Vs}$ and $\mathrm{Vm}$ in the ACA, which is similar to the results of Kim's study ${ }^{25)}$. As explained above, in the process of motor observation, the mirror neuron system of the frontal and parietal lobes is activated, which leads to understanding of the motion and ability to perform the action. Thus, it plays a role not only in accepting the external visual stimulation but also in understanding the external stimulation and in motor reaction planning as part of the information processing stage.

This study investigated whether AOT affects Vs and Vm of CBF in healthy subjects. Considering that AOT increased Vs and Vm, patients' new circumstances or activities may contribute to the positive effects of cognitive neurorehabilitation based on the performance of motor tasks. Future studies using observational training programs in stroke survivors should be conducted to further clarify the effects of AOT.

\section{REFERENCES}

1) Garrison KA, Winstein CJ, Aziz-Zadeh L: The mirror neuron system: neural substrate for methods in stroke rehabilitation. Neurorehabil Neural Repair, 2010, 24: 404-412. [Medline] [CrossRef]

2) Iacoboni M: Neural mechanisms of imitation. Curr Opin Neurobiol, 2005, 15: 632-637. [Medline] [CrossRef]

3) Kim JH, Chung EJ, Lee BH: A study of analysis of the brain wave with respected to action observation and motor imagery: a pilot randomized controlled trial. J Phys Ther Sci, 2013, 25: 779-782. [Medline] [CrossRef]

4) Kim J, Lee B, Lee HS, et al.: Differences in brain waves of normal persons and stroke patients during action observation and motor imagery. J Phys Ther Sci, 2014, 26: 215-218. [Medline] [CrossRef]

5) Lacourse MG, Orr EL, Cramer SC, et al.: Brain activation during execu- tion and motor imagery of novel and skilled sequential hand movements. Neuroimage, 2005, 27: 505-519. [Medline] [CrossRef]

6) Decety J, Grèzes J: The power of simulation: imagining one's own and other's behavior. Brain Res, 2006, 1079: 4-14. [Medline] [CrossRef]

7) Maeda F, Kleiner-Fisman G, Pascual-Leone A: Motor facilitation while observing hand actions: specificity of the effect and role of observer's orientation. J Neurophysiol, 2002, 87: 1329-1335. [Medline]

8) Smith D, Holmes P: The effect of imagery modality on golf putting performance. J Sport Exerc Psychol, 2004, 26: 385-395.

9) Oberman LM, Pineda JA, Ramachandran VS: The human mirror neuron system: a link between action observation and social skills. Soc Cogn Affect Neurosci, 2007, 2: 62-66. [Medline] [CrossRef]

10) Muthukumaraswamy SD, Johnson BW: Primary motor cortex activation during action observation revealed by wavelet analysis of the EEG. Clin Neurophysiol, 2004, 115: 1760-1766. [Medline] [CrossRef]

11) Giromini L, Porcelli P, Viglione DJ, et al.: The feeling of movement: EEG evidence for mirroring activity during the observations of static, ambiguous stimuli in the Rorschach cards. Biol Psychol, 2010, 85: 233-241. [Medline] [CrossRef]

12) Paulson OB, Hasselbalch SG, Rostrup E, et al.: Cerebral blood flow response to functional activation. J Cereb Blood Flow Metab, 2010, 30: 2-14. [Medline] [CrossRef]

13) Lecrux $\mathrm{C}$, Hamel $\mathrm{E}$ : The neurovascular unit in brain function and disease. Acta Physiol (Oxf), 2011, 203: 47-59. [Medline] [CrossRef]

14) Bakker MJ, Hofmann J, Churches OF, et al.: Cerebrovascular function and cognition in childhood: a systematic review of transcranial Doppler studies. BMC Neurol, 2014, 14: 43 [CrossRef]. [Medline]

15) Naqvi J, Yap KH, Ahmad G, et al.: Transcranial Doppler ultrasound: a review of the physical principles and major applications in critical care. Int J Vasc Med, 2013, 2013: 629378. [Medline]

16) Lohmann H, Dräger B, Müller-Ehrenberg S, et al.: Language lateralization in young children assessed by functional transcranial Doppler sonography. Neuroimage, 2005, 24: 780-790. [Medline] [CrossRef]

17) Salinet AS, Panerai RB, Robinson TG: Effects of active, passive and motor imagery paradigms on cerebral and peripheral hemodynamics in older volunteers: a functional TCD study. Ultrasound Med Biol, 2012, 38: $997-$ 1003. [Medline] [CrossRef]

18) Stroobant N, Vingerhoets G: Transcranial Doppler ultrasonography monitoring of cerebral hemodynamics during performance of cognitive tasks: a review. Neuropsychol Rev, 2000, 10: 213-231. [Medline] [CrossRef]

19) Ogoh S, Ainslie PN: Cerebral blood flow during exercise: mechanisms of regulation. J Appl Physiol 1985, 2009, 107: 1370-1380. [Medline] [CrossRef]

20) Querido JS, Sheel AW: Regulation of cerebral blood flow during exercise. Sports Med, 2007, 37: 765-782. [Medline] [CrossRef]

21) Franceschini M, Agosti M, Cantagallo A, et al.: Mirror neurons: action observation treatment as a tool in stroke rehabilitation. Eur J Phys Rehabil Med, 2010, 46: 517-523. [Medline]

22) Vingerhoets G, Stroobant N: Lateralization of cerebral blood flow velocity changes during cognitive tasks. A simultaneous bilateral transcranial Doppler study. Stroke, 1999, 30: 2152-2158. [Medline] [CrossRef]

23) Koch G, Versace V, Bonnì S, et al.: Resonance of cortico-cortical connections of the motor system with the observation of goal directed grasping movements. Neuropsychologia, 2010, 48: 3513-3520. [Medline] [CrossRef]

24) Rizzolatti G, Craighero L: The mirror-neuron system. Annu Rev Neurosci, 2004, 27: 169-192. [Medline] [CrossRef]

25) Kim SS, Kim TH, Lee BH: Effects of action observational training on cerebral hemodynamic changes of stroke survivors: a fTCD study. J Phys Ther Sci, 2014, 26: 331-334. [Medline] [CrossRef] 